\title{
A Simplified Synthesis of 3-(1-Arylsulfonylalkyl) Indoles and their Reaction with Reformatsky Reagents
}

\author{
Alessandro Palmieri and Marino Petrini*
}

Dipartimento di Scienze Chimiche, Università di Camerino, via S. Agostino, 1. I-62032 Camerino,Italy Phone: +39 0737 402253; fax: +39 0737 402297; e-mail : marino.petrini@unicam.it

\section{Supporting Information}

\section{Contents}

General Experimental Methods . . . . . . . . . . . . . . . . . . . . . S2

General Procedure for the Preparation of Arylsulfinic Acids $12 \ldots \ldots \ldots \ldots$. . . . . . . S2

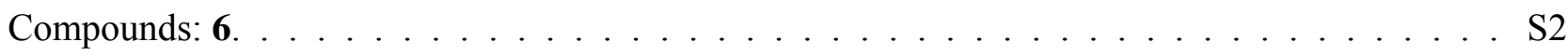

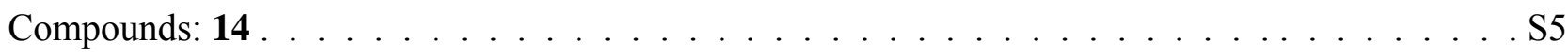

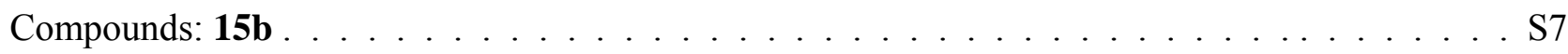




\section{General Experimental Methods}

${ }^{1} \mathrm{H}$ NMR were recorded at $400 \mathrm{MHz} .{ }^{13} \mathrm{C} \mathrm{NMR}$ were recorded at $100 \mathrm{MHz}$. GLC analyses were performed on a capillary column of fused silica $(0.32 \mathrm{~mm} \times 25 \mathrm{~m})$, stationary phase SE54. Mass spectra were performed on a GC/MS system by means of the EI technique $(70 \mathrm{eV})$. All chemicals used are commercial. Ethyl 2-indolecarboxylate 1f was prepared as previously reported. ${ }^{1}$

\section{General Procedure for the Preparation of Arylsulfinic Acids 12:}

The appropriate sodium salt $(7 \mathrm{mmol})$ was dissolved in $15 \mathrm{ml}$ of distilled water, then was added $1 \mathrm{ml}$ of concentrated sulfuric acid and after cooling at room temperature, the aqueous layer was extracted with $\mathrm{CH}_{2} \mathrm{Cl}_{2}(3 \times 20 \mathrm{~mL})$. The combined organic extracts were dried over $\mathrm{Na}_{2} \mathrm{SO}_{4}$ and after removal of the solvent at reduced pressure, the acid $\mathbf{1 2}$ was obtained in nearly quantitative yield.

\section{Spectroscopic Data for Compounds 6:}

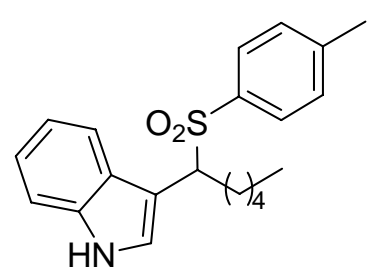

6a. Yield: $80 \%$. White solid, m.p. $106-108^{\circ} \mathrm{C}$. IR ( $\mathrm{cm}^{-1}$, nujol): 3350,1374 , 1136. ${ }^{1} \mathrm{H}-\mathrm{NMR}\left(\mathrm{CDCl}_{3}, 400 \mathrm{MHz}\right) \delta: 0.79(\mathrm{t}, 3 \mathrm{H}, J=7.9 \mathrm{~Hz}), 1.12-1.35(\mathrm{~m}$, $6 \mathrm{H}), 2.11-2.24(\mathrm{~m}, 1 \mathrm{H}), 2.31(\mathrm{~s}, 3 \mathrm{H}), 2.35-2.48(\mathrm{~m}, 1 \mathrm{H}), 4.39(\mathrm{dd}, 1 \mathrm{H}, J=3.5$, $11.5 \mathrm{~Hz}), 6.98(\mathrm{dt}, 1 \mathrm{H}, J=0.8,8.0 \mathrm{~Hz}), 7.01(\mathrm{~d}, 1 \mathrm{H}, J=2.7 \mathrm{~Hz}), 7.07(\mathrm{~d}, 2 \mathrm{H}, J$ $=7.6 \mathrm{~Hz}), 7.12(\mathrm{dt}, 1 \mathrm{H}, J=1.0,7.1 \mathrm{~Hz}), 7.25-7.35(\mathrm{~m}, 2 \mathrm{H}), 7.43(\mathrm{~d}, 2 \mathrm{H}, J=$ $8.0 \mathrm{~Hz}), 8.49$ (bs, $1 \mathrm{H}) .{ }^{13} \mathrm{C}-\mathrm{NMR}\left(\mathrm{CDCl}_{3}, 100 \mathrm{MHz}\right) \delta: 14.1,21.7,22.5,26.8$, 28.1, 31.6, 64.5, 107.5, 111.5, 119.1, 120.1, 122.3, 125.1, 127.4, 129.2, 129.3, 134.7, 136.0, 144.3. GCMS (70 eV): $m / z: 199,156$ (100), 129. Anal. Calcd. for $\mathrm{C}_{21} \mathrm{H}_{25} \mathrm{NO}_{2} \mathrm{~S}(355.49): \mathrm{C}, 70.95 ; \mathrm{H}, 7.09 ; \mathrm{N}$, 3.94. Found: C, 71.00; H, 7.01; N, 3.96.

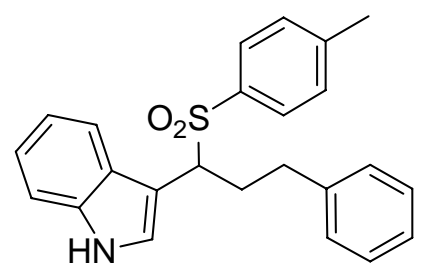

6b. Yield: $68 \%$. White solid, m.p. $151-153^{\circ} \mathrm{C} . \mathrm{IR}\left(\mathrm{cm}^{-1}\right.$, nujol): 3325,1597 , 1375, 1137. ${ }^{1} \mathrm{H}-\mathrm{NMR}\left(\mathrm{CDCl}_{3}, 400 \mathrm{MHz}\right) \delta: 2.31(\mathrm{~s}, 3 \mathrm{H}), 2.43-2.58(\mathrm{~m}$, $2 \mathrm{H}), 2.65-2.81(\mathrm{~m}, 2 \mathrm{H}), 4.38(\mathrm{dd}, 1 \mathrm{H}, J=3.0,11.1 \mathrm{~Hz}), 6.98-7.09(\mathrm{~m}, 6 \mathrm{H})$, $7.14(\mathrm{dt}, 1 \mathrm{H}, J=0.9,7.6 \mathrm{~Hz}), 7.17-7.27(\mathrm{~m}, 3 \mathrm{H}), 7.28-7.33(\mathrm{~m}, 2 \mathrm{H}), 7.41$ $(\mathrm{d}, 2 \mathrm{H}, J=8.1 \mathrm{~Hz}), 8.60(\mathrm{bs}, 1 \mathrm{H}) .{ }^{13} \mathrm{C}-\mathrm{NMR}\left(\mathrm{CDCl}_{3}, 100 \mathrm{MHz}\right) \delta: 21.8$, 29.9, 33.0, 63.7, 106.9, 111.7, 119.2, 120.2, 122.5, 125.5, 126.5, 127.3, 128.7, 128.8, 129.2, 129.4, 134.6, 136.2, 140.6, 144.5. GC-MS (70 eV): m/z: 233 (100), 217, 206, 156, 130, 115. Anal. Calcd. for $\mathrm{C}_{24} \mathrm{H}_{23} \mathrm{NO}_{2} \mathrm{~S}$ (389.51): C, 74.01; H, 5.95; N, 3.60. Found: C, 73.98; H, 5.91; N, 3.65.

\footnotetext{
${ }^{1}$ G. P. Fagan, C. B. Chapleo, A. C. Lane, M. Myers, A. G. Roach, C. F. C. Smith, M. R. Stillings, and
} A. P. Welbourn, J. Med. Chem. 1988, 31, 944. 


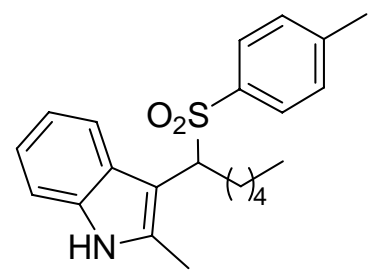

6c. Yield: $75 \%$. White solid, m.p. $134-136^{\circ} \mathrm{C} . \mathrm{IR}\left(\mathrm{cm}^{-1}\right.$, nujol): 3347,1376 , 1140. ${ }^{1} \mathrm{H}-\mathrm{NMR}\left(\mathrm{CDCl}_{3}, 400 \mathrm{MHz}\right) \delta: 0.75-0.81(\mathrm{~m}, 3 \mathrm{H}), 1.10-1.31(\mathrm{~m}, 6 \mathrm{H})$, $1.85(\mathrm{~s}, 3 \mathrm{H}), 2.34(\mathrm{~s}, 3 \mathrm{H}), 2.20-2.66(\mathrm{~m}, 2 \mathrm{H}), 4.20(\mathrm{dd}, 1 \mathrm{H}, J=3.2,11.0 \mathrm{~Hz})$, 6.97-7.14 (m, 4H), $7.21(\mathrm{~d}, 1 \mathrm{H}, J=8.0 \mathrm{~Hz}), 7.36(\mathrm{~d}, 2 \mathrm{H}, J=7.4 \mathrm{~Hz}), 7.63(\mathrm{~d}$, $1 \mathrm{H}, J=7.9 \mathrm{~Hz}), 8.08$ (bs, $1 \mathrm{H}) .{ }^{13} \mathrm{C}-\mathrm{NMR}\left(\mathrm{CDCl}_{3}, 100 \mathrm{MHz}\right) \delta: 11.4,14.1$, $21.7,22.5,25.8,27.0,31.5,65.8,102.9,110.6,120.0,120.9,121.4,126.7$, 129.0, 129.7, 135.2, 135.4, 136.2, 144.2. GC-MS (70 eV): $m / z: 213,170$ (100), 154, 144, 129. Anal. Calcd. for $\mathrm{C}_{22} \mathrm{H}_{27} \mathrm{NO}_{2} \mathrm{~S}$ (369.52): C, 71.51; H, 7.36; N, 3.79. Found: C, 71.56; H, 7.32; N, 3.80.

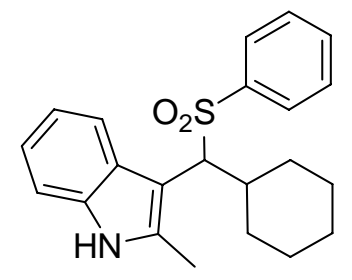

6d. Yield $82 \%$. White solid, m.p. $150-152^{\circ} \mathrm{C}$. IR ( $\mathrm{cm}^{-1}$, nujol): $3334,1376,1130$. ${ }^{1} \mathrm{H}-\mathrm{NMR}\left(\mathrm{CDCl}_{3}, 400 \mathrm{MHz}\right) \delta:$ 0.78-0.94 (m, 1H), 1.08-1.31 (m, 2H), 1.34-1.71 $(\mathrm{m}, 5 \mathrm{H}), 1.78(\mathrm{~s}, 2.55 \mathrm{H}), 1.75-1.88(\mathrm{~m}, 1 \mathrm{H}), 2.61(\mathrm{~s}, 0.45 \mathrm{H}), 2.67-2.80(\mathrm{~m}, 1 \mathrm{H})$, $2.90-3.03(\mathrm{~m}, 1 \mathrm{H}), 4.13(\mathrm{~d}, 0.85 \mathrm{H}, J=9.8 \mathrm{~Hz}), 4.42(\mathrm{~d}, 0.15 \mathrm{H}, J=9.8 \mathrm{~Hz})$, 6.75-7.06 (m, 1H), 7.08-7.21 (m, 4H), 7.38-7.29 (m, 2.85H), 7.46 (d, 0.15H, $J=$ $7.3 \mathrm{~Hz}), 7.78$ (bs, $1 \mathrm{H}), 7.93-8.00(\mathrm{~m}, 1 \mathrm{H}) .{ }^{13} \mathrm{C}-\mathrm{NMR}\left(\mathrm{CDCl}_{3}, 100 \mathrm{MHz}\right) \delta: 11.4$, 14.4, 26.0, 26.1, 26.4, 26.5, 31.7, 31.9, 33.0, 33.2, 36.9, 37.4, 69.2, 71.4, 104.5, 110.1, 110.4, 116.6, 119.5, 120.3, 121.3, 121.6, 121.7, 126.7, 128.0, 128.1, 128.2, 132.5, 132.9, 135.3, 135.4, 140.0. GCMS (70 eV): m/z: 225 (100), 210, 196, 182, 168, 144, 130. Anal. Calcd. for $\mathrm{C}_{22} \mathrm{H}_{25} \mathrm{NO}_{2} \mathrm{~S}$ (367.5): C, 71.90; H, 6.86; N, 3.81. Found: C, 71.95; H, 6.90; N, 3.79 .

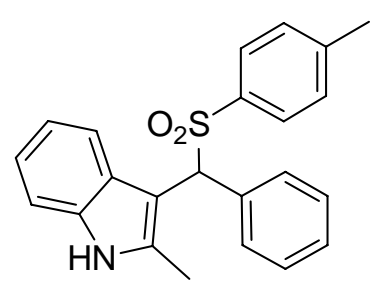

6f. Yield 95\%. White solid, m.p. 80-83 ${ }^{\circ} \mathrm{C}$. IR ( $\mathrm{cm}^{-1}$, nujol): $3349,1596,1376$, 1139. ${ }^{1} \mathrm{H}-\mathrm{NMR}\left(\mathrm{CDCl}_{3}, 400 \mathrm{MHz}\right) \delta: 2.03(\mathrm{~s}, 3 \mathrm{H}), 2.31(\mathrm{~s}, 3 \mathrm{H}), 5.63(\mathrm{~s}, 1 \mathrm{H})$, 7.01-7.15 (m, 4H), $7.20(\mathrm{~d}, 1 \mathrm{H}, J=7.3 \mathrm{~Hz}), 7.27-7.36(\mathrm{~m}, 3 \mathrm{H}), 7.37-7.46(\mathrm{~m}$, 2H), 7.69-7.85 (m, 3H), 7.97 (bs, $1 \mathrm{H}) .{ }^{13} \mathrm{C}-\mathrm{NMR}\left(\mathrm{CDCl}_{3}, 100 \mathrm{MHz}\right) \delta: 12.2$, $21.8,69.8,104.6,110.5,120.3,121.4,121.8,128.4,128.7,128.9,129.2$, 129.9, 130.2, 133.3, 135.3, 135.5, 136.2, 144.3. GC-MS (70 eV) $\mathrm{m} / z: 155,139$ , 91 (100), 77. Anal. Calcd. for $\mathrm{C}_{23} \mathrm{H}_{21} \mathrm{NO}_{2} \mathrm{~S}$ (375.48): C, 73.57; H, 5.64; N, 3.73. Found: C, 73.55; H, $5.68 ; \mathrm{N}, 3.74$.

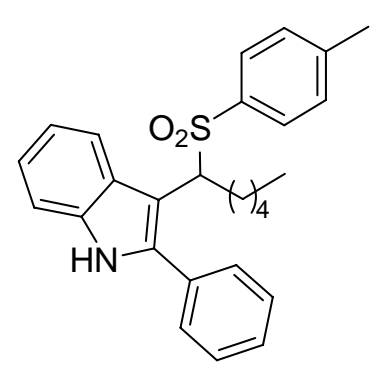

6g. Yield 67\%. White solid, m.p. 94-96 ${ }^{\circ} \mathrm{C}$. IR ( $\mathrm{cm}^{-1}$, nujol): 3338, 1596, 1377, 1140. ${ }^{1} \mathrm{H}-\mathrm{NMR}\left(\mathrm{CDCl}_{3}, 400 \mathrm{MHz}\right) \delta: 0.75-0.90(\mathrm{~m}, 3 \mathrm{H}), 1.15-1.27(\mathrm{~m}, 6 \mathrm{H})$, $2.36(\mathrm{~s}, 3 \mathrm{H}), 2.29-2.47(\mathrm{~m}, 1 \mathrm{H}), 2.56-2.72(\mathrm{~m}, 1 \mathrm{H}), 4.44(\mathrm{dd}, 1 \mathrm{H}, J=3.5,11.4$ $\mathrm{Hz}), 7.01-7.10(\mathrm{~m}, 4 \mathrm{H}), 7.16(\mathrm{t}, 1 \mathrm{H}, J=7.5 \mathrm{~Hz}), 7.23(\mathrm{t}, 1 \mathrm{H}, J=7.5 \mathrm{~Hz})$, $7.32-7.41(\mathrm{~m}, 4 \mathrm{H}), 7.96(\mathrm{~d}, 1 \mathrm{H}, J=7.8 \mathrm{~Hz}), 8.13(\mathrm{bs}, 1 \mathrm{H}) .{ }^{13} \mathrm{C}-\mathrm{NMR}\left(\mathrm{CDCl}_{3}\right.$, $100 \mathrm{MHz}) \delta: 13.9,21.6,22.3,26.1,26.9,31.4,65.1,104.2,110.9,120.4$, $122.3,122.6,126.5,128.5,128.7,128.8,129.1,131.5,135.4,135.9,139.6$, 143.8. GC-MS (70 eV) m/z: 275, 232 (100), 217. Anal. Calcd. for $\mathrm{C}_{27} \mathrm{H}_{29} \mathrm{NO}_{2} \mathrm{~S}$ (431.59): C, 75.14; $\mathrm{H}$, 6.77; N, 3.25. Found: C, 75.18; H, 6.74; N, 3.27. 


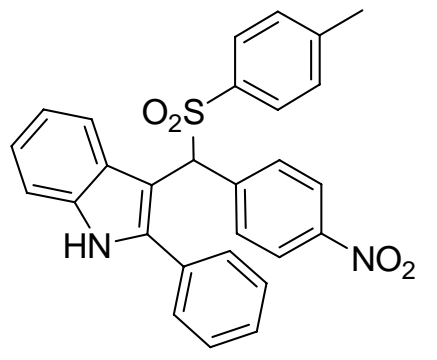

6h. Yield $81 \%$. Yellow solid, m.p. $120^{\circ} \mathrm{C}$ (dec.). IR ( $\mathrm{cm}^{-1}$, nujol): 3387 , 1592, 1517, 1377, 1347, 1114. ${ }^{1} \mathrm{H}-\mathrm{NMR}\left(\mathrm{CDCl}_{3}, 400 \mathrm{MHz}\right) \delta: 2.35$ (s, 3H), $5.84(\mathrm{~s}, 1 \mathrm{H}), 6.93(\mathrm{~d}, 2 \mathrm{H}, J=7.7 \mathrm{~Hz}), 6.98(\mathrm{~d}, 2 \mathrm{H}, J=8.1 \mathrm{~Hz}), 7.19(\mathrm{~d}, 2 \mathrm{H}$, $J=8.1 \mathrm{~Hz}), 7.21-7.30(\mathrm{~m}, 2 \mathrm{H}), 7.32-7.45(\mathrm{~m}, 4 \mathrm{H}), 8.00(\mathrm{~d}, 2 \mathrm{H}, J=8.5$ $\mathrm{Hz}), 8.12(\mathrm{~d}, 1 \mathrm{H}, J=7.7 \mathrm{~Hz}), 8.17-8.23(\mathrm{~m}, 3 \mathrm{H}) .{ }^{13} \mathrm{C}-\mathrm{NMR}\left(\mathrm{CDCl}_{3}, 100\right.$ MHz) $\delta: 21.8,69.6,104.1,111.3,121.4,123.2,123.3,123.8,126.6,128.6$, $128.9,129.1,129.3,129.4,131.1,131.4,135.4,136.1,140.1,140.6,144.6$, 147.9. Anal. Calcd. for $\mathrm{C}_{28} \mathrm{H}_{22} \mathrm{~N}_{2} \mathrm{O}_{4} \mathrm{~S}$ (482.55): C, 69.69; H, 4.60; N, 5.81. Found: C, 69.65; H, 4.63; N, 5.83.

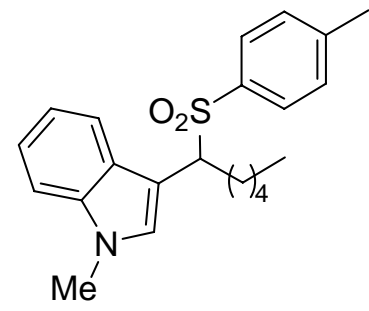

6i. Yield $80 \%$. Waxy solid. IR ( $\mathrm{cm}^{-1}$, neat): 3116, 3051, 1614, 1597, 1140. ${ }^{1} \mathrm{H}-\mathrm{NMR}\left(\mathrm{CDCl}_{3}, 400 \mathrm{MHz}\right) \delta: 0.80(\mathrm{t}, 3 \mathrm{H}, J=7.3 \mathrm{~Hz}), 1.14-1.37(\mathrm{~m}, 6 \mathrm{H})$, 2.09-2.23 (m, 1H), $2.31(\mathrm{~s}, 3 \mathrm{H}), 2.35-2.46(\mathrm{~m}, 1 \mathrm{H}), 3.75(\mathrm{~s}, 3 \mathrm{H}), 4.37(\mathrm{dd}, 1 \mathrm{H}$, $J=3.5,11.7 \mathrm{~Hz}), 6.95-7.00(\mathrm{~m}, 2 \mathrm{H}), 7.07(\mathrm{dd}, 2 \mathrm{H}, J=0.5,8.5 \mathrm{~Hz}), 7.13-7.19$ $(\mathrm{m}, 1 \mathrm{H}), 7.26(\mathrm{t}, 2 \mathrm{H}, J=7.5 \mathrm{~Hz}), 7.44(\mathrm{~d}, 2 \mathrm{H}, J=8.5 \mathrm{~Hz}) .{ }^{13} \mathrm{C}-\mathrm{NMR}\left(\mathrm{CDCl}_{3}\right.$, $100 \mathrm{MHz}) \delta: 14.1,21.6,22.5,26.8,28.2,31.6,33.2,64.4,105.9,109.4,119.1$, 119.7, 121.9, 128.0, 129.1, 129.2, 129.4, 134.9, 136.8, 144.2. GC-MS (70 eV) m/z: 213, 170 (100), 154, 144, 128, 42. Anal. Calcd. for $\mathrm{C}_{22} \mathrm{H}_{27} \mathrm{NO}_{2} \mathrm{~S}$ (369.52): C, 71.51; H, 7.36; N, 3.79. Found: C, 71.54; H, 7.33; N, 3.77 .

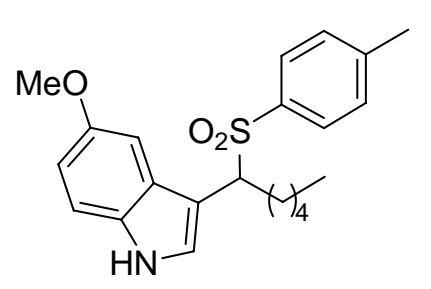

6j. Yield $79 \%$. White solid, m.p. $132-134^{\circ} \mathrm{C}$. IR ( $\mathrm{cm}^{-1}$, nujol): 3342,1623 , 1377, 1134. ${ }^{1} \mathrm{H}-\mathrm{NMR}\left(\mathrm{CDCl}_{3}, 400 \mathrm{MHz}\right) \delta: 0.80(\mathrm{t}, 3 \mathrm{H}, J=7.4 \mathrm{~Hz}), 1.14$ $1.38(\mathrm{~m}, 6 \mathrm{H}), 2.07-2.22(\mathrm{~m}, 1 \mathrm{H}), 2.31(\mathrm{~s}, 3 \mathrm{H}), 2.38-2.49(\mathrm{~m}, 1 \mathrm{H}), 3.73(\mathrm{~s}$, $3 \mathrm{H}), 4.34(\mathrm{dd}, 1 \mathrm{H}, J=3.5,11.7 \mathrm{~Hz}), 6.66(\mathrm{~d}, 1 \mathrm{H}, J=2.3 \mathrm{~Hz}), 6.76(\mathrm{dd}, 1 \mathrm{H}$, $J=2.3,9.0 \mathrm{~Hz}), 6.99(\mathrm{~d}, 1 \mathrm{H}, J=2.7 \mathrm{~Hz}), 7.07(\mathrm{~d}, 2 \mathrm{H}, J=8.2 \mathrm{~Hz}), 7.17(\mathrm{~d}$, $1 \mathrm{H}, J=8.6 \mathrm{~Hz}), 7.42(\mathrm{~d}, 2 \mathrm{H}, J=8.6 \mathrm{~Hz}), 8.45(\mathrm{bs}, 1 \mathrm{H}) .{ }^{13} \mathrm{C}-\mathrm{NMR}\left(\mathrm{CDCl}_{3}\right.$, $100 \mathrm{MHz}) \delta: 14.1,21.6,22.5,26.8,27.9,31.6,55.8,64.7,100.5,107.1,112.2,112.7,125.7,127.9$, 129.3, 131.1, 134.7, 144.3, 154.3. GC-MS (70 eV) m/z: 278, 246, 155, 139 (100), 123, 91, 77, 65, 45. Anal. Calcd. for $\mathrm{C}_{22} \mathrm{H}_{27} \mathrm{NO}_{3} \mathrm{~S}$ (385.52): C, 68.54; H, 7.06; N, 3.63. Found: C, 68.57; H, 7.04; N, 3.61.

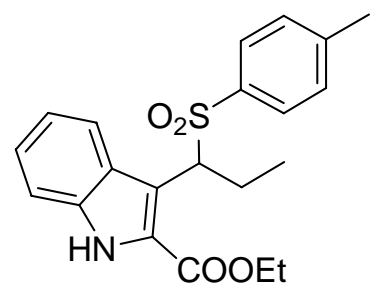

6k. Yield $71 \%$. White solid, m.p. $161-163^{\circ} \mathrm{C}$. IR $\left(\mathrm{cm}^{-1}\right.$, nujol): 3310,1702 , 1193, $1139 .{ }^{1} \mathrm{H}-\mathrm{NMR}\left(\mathrm{CD}_{3} \mathrm{COCD}_{3}, 400 \mathrm{MHz}\right) \delta: 0.84(\mathrm{t}, 3 \mathrm{H}, J=7.7 \mathrm{~Hz})$, $1.29(\mathrm{t}, 3 \mathrm{H}, J=7.3 \mathrm{~Hz}), 2.35(\mathrm{~s}, 3 \mathrm{H}), 2.41-2.65(\mathrm{~m}, 2 \mathrm{H}), 4.14-4.31(\mathrm{~m}, 2 \mathrm{H})$, $5.65(\mathrm{dd}, 1 \mathrm{H}, J=11.5,4.3 \mathrm{~Hz}), 7.12-7.17(\mathrm{~m}, 1 \mathrm{H}), 7.22(\mathrm{~d}, 2 \mathrm{H}, J=7.7 \mathrm{~Hz})$, $7.29-7.34(\mathrm{~m}, 1 \mathrm{H}), 7.40(\mathrm{~d}, 2 \mathrm{H}, J=8.1 \mathrm{~Hz}), 7.49(\mathrm{~d}, 1 \mathrm{H}, J=8.1 \mathrm{~Hz}), 8.05(\mathrm{~d}$, $1 \mathrm{H}, J=8.1 \mathrm{~Hz}), 10.94$ (bs, $1 \mathrm{H}) .{ }^{13} \mathrm{C}-\mathrm{NMR}\left(\mathrm{CD}_{3} \mathrm{COCD}_{3}, 100 \mathrm{MHz}\right) \delta: 12.2$, 14.6, 20.7, 21.5, 61.5, 65.6, 113.4, 113.5, 121.3, 124.5, 126.1, 126.8, 127.6, 129.5, 129.9, 137.0, 137.4, 145.0, 161.9. GC-MS (70 eV) m/z: 229, 183, 154 (100), 128. Anal. Calcd. for $\mathrm{C}_{21} \mathrm{H}_{23} \mathrm{NO}_{4} \mathrm{~S}$ (385.48): C, 65.43; H, 6.01; N, 3.63. Found: C, 65.47; H, 5.98; N, 3.61. 


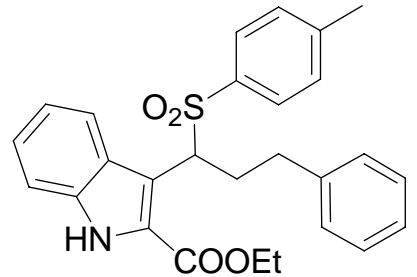

6l. Yield: $75 \%$. White solid, m.p. $157-159^{\circ} \mathrm{C}$. IR ( $\mathrm{cm}^{-1}$, nujol): 3311,1708 , 1186, 1140. ${ }^{1} \mathrm{H}-\mathrm{NMR}\left(\mathrm{CDCl}_{3}, 400 \mathrm{MHz}\right) \delta: 1.20(\mathrm{t}, 3 \mathrm{H}, J=7.3 \mathrm{~Hz}), 2.34(\mathrm{~s}$, $3 \mathrm{H}), 2.35-2.46(\mathrm{~m}, 1 \mathrm{H}), 2.55-2.67(\mathrm{~m}, 1 \mathrm{H}), 2.74-2.86(\mathrm{~m}, 1 \mathrm{H}), 2.90-3.03$ $(\mathrm{m}, 1 \mathrm{H}), 3.95-4.15(\mathrm{~m}, 2 \mathrm{H}), 5.59(\mathrm{dd}, 1 \mathrm{H}, J=11.1,3.8 \mathrm{~Hz}), 6.96(\mathrm{~d}, 2 \mathrm{H}, J=$ $7.7 \mathrm{~Hz}), 7.10(\mathrm{~d}, 2 \mathrm{H}, J=8.1 \mathrm{~Hz}), 7.12-7.27(\mathrm{~m}, 4 \mathrm{H}), 7.33-7.46(\mathrm{~m}, 4 \mathrm{H})$, $8.12(\mathrm{~d}, 1 \mathrm{H}, J=8.1 \mathrm{~Hz}), 9.02(\mathrm{bs}, 1 \mathrm{H}) .{ }^{13} \mathrm{C}-\mathrm{NMR}\left(\mathrm{CDCl}_{3}, 100 \mathrm{MHz}\right) \delta$ : $14.3,21.7,27.9,33.1,61.2,63.0,112.2,113.4,121.5,124.0,126.0,126.2,126.3,126.5,128.4,128.5$, 128.9, 129.1, 135.2, 135.9, 140.4, 144.3, 161.1. GC-MS (70 eV) m/z: 305, 258 (100), 230, 214, 186, 154, 128, 115, 91, 77, 29. Anal. Calcd. for $\mathrm{C}_{27} \mathrm{H}_{27} \mathrm{NO}_{4} \mathrm{~S}$ (461.57): C, 70.26; H, 5.90; N, 3.03. Found: C, $70.30 ; \mathrm{H}, 5.88 ; \mathrm{N}, 3.00$.

\section{Spectroscopic Data for Compounds 14.}<smiles>COC(=O)CC(C)c1c[nH]c2ccccc12</smiles>

14a. Yield $80 \%$. Oil. IR ( $\mathrm{cm}^{-1}$, neat) $3371,3051,1735 .{ }^{1} \mathrm{H}-\mathrm{NMR}\left(\mathrm{CDCl}_{3}, 400\right.$

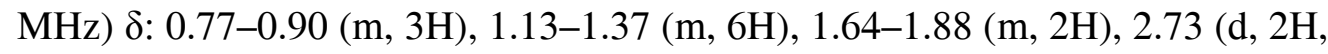
$J=7.7 \mathrm{~Hz}), 3.41-3.50(\mathrm{~m}, 1 \mathrm{H}), 3.59$ (s, 3H), 6.99 (s, 1H), 7.08-7.14 (m, 1H), 7.16-7.21 (m, 1H), $7.35(\mathrm{~d}, 1 \mathrm{H}, J=8.1 \mathrm{~Hz}), 7.66(\mathrm{~d}, 1 \mathrm{H}, J=7.7 \mathrm{~Hz}), 8.00(\mathrm{bs}$, 1H). ${ }^{13} \mathrm{C}-\mathrm{NMR}\left(\mathrm{CDCl}_{3}, 100 \mathrm{MHz}\right) \delta: 14.3,22.8,27.4,32.1,33.8,35.7,41.3$, 51.7, 111.4, 119.2, 119.4, 119.6, 121.2, 122.1, 126.9, 136.7, 173.7. GC-MS (70 eV) m/z : 273 (100), 202, 200, 160, 143, 130, 115. Anal. Calcd. for $\mathrm{C}_{17} \mathrm{H}_{23} \mathrm{NO}_{2}$ (273.37): C, 74.69; H, 8.48; N, 5.12. Found: C, $74.74 ; \mathrm{H}, 8.45 ; \mathrm{N}, 5.15$.

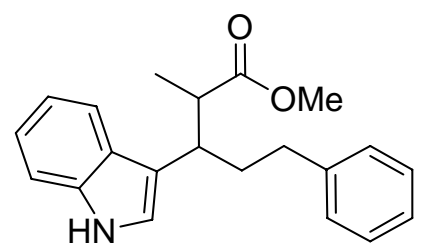

14b. (Diastereomer A) Yield 45\%. Oil. IR ( $\mathrm{cm}^{-1}$, neat) 3417, 3060, 3026, 1731, 1196. ${ }^{1} \mathrm{H}-\mathrm{NMR}\left(\mathrm{CDCl}_{3}, 400 \mathrm{MHz}\right) \delta: 1.01(\mathrm{~d}, 3 \mathrm{H}, J=7.3 \mathrm{~Hz}), 1.93-$ $2.04(\mathrm{~m}, 1 \mathrm{H}), 2.10-2.22(\mathrm{~m}, 1 \mathrm{H}), 2.37-2.56(\mathrm{~m}, 2 \mathrm{H}), 2.87-2.98(\mathrm{~m}, 1 \mathrm{H})$, 3.15-3.24 (m, 1H), $3.69(\mathrm{~s}, 3 \mathrm{H}), 7.02(\mathrm{~d}, 1 \mathrm{H}, J=2.6 \mathrm{~Hz}), 7.04-7.08(\mathrm{~m}$, 2H), 7.10-7.18 (m, 2H), 7.20-7.27 (m, 3H), 7.39 (d, 1H, $J=8.1 \mathrm{~Hz}), 7.67$ (dd, $1 \mathrm{H}, J=7.7,1.3 \mathrm{~Hz}), 8.13$ (bs, $1 \mathrm{H}) .{ }^{13} \mathrm{C}-\mathrm{NMR}\left(\mathrm{CDCl}_{3}, 100 \mathrm{MHz}\right) \delta: 16.3,34.3,36.0,40.6,45.6$, $51.8,111.5,116.3,119.5,119.7,122.2,122.6,125.8,127.2,128.4,128.6,136.8,142.5,177.4$. GC-MS (70 eV) m/z: 321, 234, 156, 130, 117, 91 (100), 65. Anal. Calcd. for $\mathrm{C}_{21} \mathrm{H}_{23} \mathrm{NO}_{2}$ (321.41): C, 78.47; $\mathrm{H}$, 7.21; N, 4.36. Found: C, 78.42; H, 7.18; N, 4.33. (Diastereomer B) Yield 30\%. Oil. IR ( $\mathrm{cm}^{-1}$, neat) 3410, 3055, 3031, 1733, 1201. ${ }^{1} \mathrm{H}-\mathrm{NMR}\left(\mathrm{CDCl}_{3}, 400 \mathrm{MHz}\right) \delta: 1.17(\mathrm{~d}, 3 \mathrm{H}, J=7.3 \mathrm{~Hz}), 2.04-2.14(\mathrm{~m}$, 2H), 2.41-2.43 (m, 1H), 2.56-2.66 (m, 1H), 2.86-2.96 (m, 1H), 3.39 (q, 1H, J = 7.3 Hz), 3.49 (s, 3H), 6.99-7.30 (m, 8H), $7.38(\mathrm{~d}, 1 \mathrm{H}, J=8.1 \mathrm{~Hz}), 7.65(\mathrm{~d}, 1 \mathrm{H}, J=7.7 \mathrm{~Hz}), 8.10$ (bs, $1 \mathrm{H}) .{ }^{13} \mathrm{C}-\mathrm{NMR}\left(\mathrm{CDCl}_{3}\right.$, $100 \mathrm{MHz}) \delta: 13.9,33.1,34.0,39.0,45.1,51.6,111.3,117.3,119.4,121.9,122.1,125.9,127.4,128.4$, 128.5, 136.5, 142.6, 176.5. Anal. Calcd. for $\mathrm{C}_{21} \mathrm{H}_{23} \mathrm{NO}_{2}$ (321.41): C, 78.47; H, 7.21; N, 4.36. Found: C, $78.50 ; \mathrm{H}, 7.20 ; \mathrm{N}, 4.38$. 


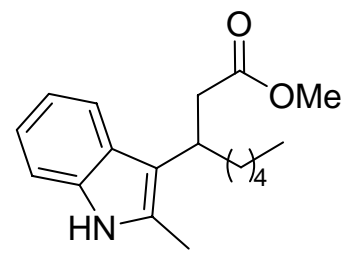

14c. Yield 90\%. Oil. IR (cm ${ }^{-1}$, neat) $3401,3055,3024,1731 .{ }^{1} \mathrm{H}-\mathrm{NMR}\left(\mathrm{CDCl}_{3}\right.$, $400 \mathrm{MHz}) \delta$ : 0.78-0.85 (m, 3H), 1.08-1.30 (m, 6H), 1.66-1.77 (m, 1H), 1.85$1.98(\mathrm{~m}, 1 \mathrm{H}), 2.39(\mathrm{~s}, 3 \mathrm{H}), 2.81(\mathrm{~d}, 2 \mathrm{H}, J=7.7 \mathrm{~Hz}), 3.30-3.40(\mathrm{~m}, 1 \mathrm{H}), 3.56(\mathrm{~s}$, $3 \mathrm{H}), 7.01-7.12(\mathrm{~m}, 2 \mathrm{H}), 7.25(\mathrm{~d}, 1 \mathrm{H}, J=7.3 \mathrm{~Hz}), 7.57(\mathrm{~d}, 1 \mathrm{H}, J=7.3 \mathrm{~Hz}), 7.73$ (bs, 1H). ${ }^{13} \mathrm{C}-\mathrm{NMR}\left(\mathrm{CDCl}_{3}, 100 \mathrm{MHz}\right) \delta: 12.4,14.3,22.8,27.9,32.0,33.8,34.9$, 40.6, 51.6, 110.6, 113.3, 119.1, 119.3, 120.8, 127.4, 131.7, 135.8, 173.8. GC-MS (70 eV) m/z : 287, 216 (100), 214, 174, 157, 144. Anal. Calcd. for $\mathrm{C}_{18} \mathrm{H}_{25} \mathrm{NO}_{2}$ (287.4): C, 75.22; H, 8.77; N, 4.87. Found: C, 75.27; H, 8.79; N, 4.90.

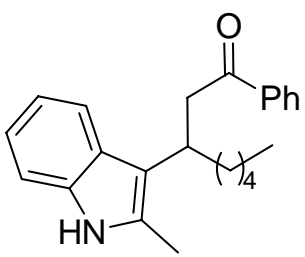

14e. Yield: $88 \%$. Oil. IR ( $\mathrm{cm}^{-1}$, neat): $3395,3026,1710 .{ }^{1} \mathrm{H}-\mathrm{NMR}\left(\mathrm{CDCl}_{3}, 400\right.$

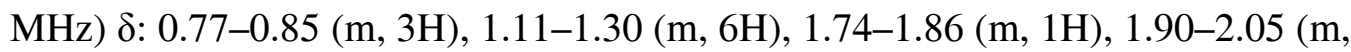
$1 \mathrm{H}), 2.36(\mathrm{~s}, 3 \mathrm{H}), 3.31-3.41(\mathrm{~m}, 1 \mathrm{H}), 3.45-3.61(\mathrm{~m}, 2 \mathrm{H}), 7.03-7.12(\mathrm{~m}, 2 \mathrm{H})$, 7.22-7.27 (m, 1H), $7.36(\mathrm{t}, 2 \mathrm{H}, J=7.7 \mathrm{~Hz}), 7.45-7.51(\mathrm{~m}, 1 \mathrm{H}), 7.64-7.68(\mathrm{~m}$, $1 \mathrm{H}), 7.71(\mathrm{bs}, 1 \mathrm{H}), 7.85(\mathrm{dd}, 2 \mathrm{H}, J=8.1,1.3 \mathrm{~Hz}) .{ }^{13} \mathrm{C}-\mathrm{NMR}\left(\mathrm{CDCl}_{3}, 100 \mathrm{MHz}\right) \delta$ : 12.2, 14.3, 22.8, 28.0, 32.0, 33.0, 35.2, 44.8, 110.6, 113.9, 119.0, 119.2, 120.7, 127.4, 128.2, 128.6, 131.6, 132.9, 135.8, 137.5, 200.4. GC-MS (70 eV) m/z: 333, 262, 214, 144, 105 (100), 77. Anal. Calcd. for $\mathrm{C}_{23} \mathrm{H}_{27} \mathrm{NO}$ (333.47): C, 82.84; H, 8.16; N, 4.20. Found: 82.80; H, 8.13; N, 4.18.

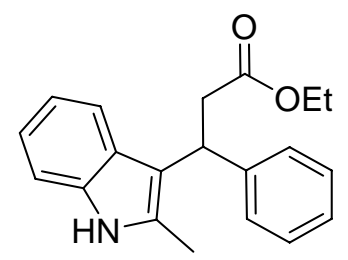

14f. Yield $66 \%$. Oil. IR ( $\mathrm{cm}^{-1}$, neat) $3380,3049,1721 .{ }^{1} \mathrm{H}-\mathrm{NMR}\left(\mathrm{CDCl}_{3}, 400\right.$ MHz) $\delta: 1.08(\mathrm{t}, 3 \mathrm{H}, J=7.3 \mathrm{~Hz}), 2.40(\mathrm{~s}, 3 \mathrm{H}), 3.20(\mathrm{dd}, 1 \mathrm{H}, J=15.4,8.7 \mathrm{~Hz})$, $3.32(\mathrm{dd}, 1 \mathrm{H}, J=15.4,6.8 \mathrm{~Hz}), 4.01(\mathrm{q}, 2 \mathrm{H}, J=7.3 \mathrm{~Hz}), 4.81-4.88(\mathrm{~m}, 1 \mathrm{H})$, 6.98-7.03 (m, 1H), $7.07(\mathrm{dt}, 1 \mathrm{H}, J=7.7,1.3 \mathrm{~Hz}), 7.13-7.19(\mathrm{~m}, 1 \mathrm{H}), 7.21-7.29$ $(\mathrm{m}, 3 \mathrm{H}), 7.32-7.37(\mathrm{~m}, 2 \mathrm{H}), 7.47(\mathrm{~d}, 1 \mathrm{H}, J=7.7 \mathrm{~Hz}), 7.78(\mathrm{bs}, 1 \mathrm{H}) .{ }^{13} \mathrm{C}-\mathrm{NMR}$ $\left(\mathrm{CDCl}_{3}, 100 \mathrm{MHz}\right) \delta: 12.5,14.3,38.1,39.8,60.6,110.5,113.4,119.3,119.4$, 121.0, 126.2, 127.6, 127.7, 128.5, 131.8, 135.6, 143.9, 172.7. GC-MS (70 eV) m/z: 307, 220 (100), 204, 178, 130. Anal. Calcd. for $\mathrm{C}_{20} \mathrm{H}_{21} \mathrm{NO}_{2}$ (307.39): C, 78.15; H, 6.89; N, 4.56. Found: C, 78.20; H, $6.93 ; \mathrm{N}, 4.59$.

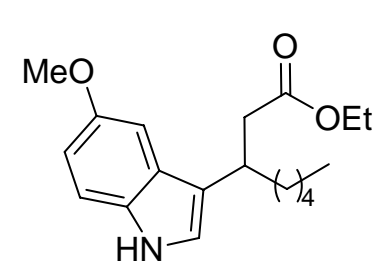

14g. Yield 67\%. Oil. IR ( $\mathrm{cm}^{-1}$, neat) $3410,1715,1214 .{ }^{1} \mathrm{H}-\mathrm{NMR}\left(\mathrm{CDCl}_{3}, 400\right.$ MHz) $\delta: 0.79-0.87(\mathrm{~m}, 3 \mathrm{H}), 1.14(\mathrm{t}, 3 \mathrm{H}, J=7.3 \mathrm{~Hz}), 1.18-1.32(\mathrm{~m}, 6 \mathrm{H})$, $1.61-1.84(\mathrm{~m}, 2 \mathrm{H}), 2.69(\mathrm{~d}, 2 \mathrm{H}, J=7.7 \mathrm{~Hz}), 3.36-3.46(\mathrm{~m}, 1 \mathrm{H}), 3.87(\mathrm{~s}, 3 \mathrm{H})$, $4.05(\mathrm{q}, 2 \mathrm{H}, J=7.3 \mathrm{~Hz}), 6.84(\mathrm{dd}, 1 \mathrm{H}, J=8.5,2.6 \mathrm{~Hz}), 6.96(\mathrm{~d}, 1 \mathrm{H}, J=2.6$ $\mathrm{Hz}), 7.09(\mathrm{~d}, 1 \mathrm{H}, J=2.6 \mathrm{~Hz}), 7.22(\mathrm{~d}, 1 \mathrm{H}, J=8.5 \mathrm{~Hz}), 7.95(\mathrm{bs}, 1 \mathrm{H}) .{ }^{13} \mathrm{C}-$ NMR $\left(\mathrm{CDCl}_{3}, 100 \mathrm{MHz}\right) \delta: 14.2,14.3,22.8,27.3,32.0,33.6,35.6,41.4$, 56.2, 60.4, 101.6, 111.9, 112.0, 118.9, 122.0, 127.4, 131.8, 153.8, 173.3. GC-MS (70 eV) m/z: 317, 246, 230, 160 (100), 130, 29. Anal. Calcd. for $\mathrm{C}_{19} \mathrm{H}_{27} \mathrm{NO}_{3}$ (317.42): C, 71.89; H, 8.57; N, 4.41. Found: C, $71.85 ; \mathrm{H}, 8.59 ; \mathrm{N}, 4.44$. 


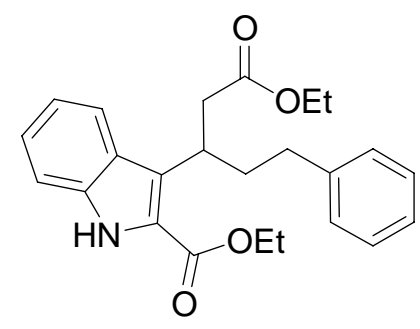

15b Yield 60\%. Oil. IR ( $\mathrm{cm}^{-1}$, neat): 3381, 3060, 3023, 1716. ${ }^{1} \mathrm{H}-\mathrm{NMR}$ $\left(\mathrm{CDCl}_{3}, 400 \mathrm{MHz}\right) \delta: 1.09(\mathrm{t}, 3 \mathrm{H}, J=7.3 \mathrm{~Hz}), 1.40(\mathrm{t}, 3 \mathrm{H}, J=7.3 \mathrm{~Hz})$, 2.11-2.23 (m, 1H), 2.29-2.59 (m, 3H), $2.92(\mathrm{~d}, 2 \mathrm{H}, J=7.7 \mathrm{~Hz}), 3.93-4.06$ $(\mathrm{m}, 2 \mathrm{H}), 4.25-4.46(\mathrm{~m}, 3 \mathrm{H}), 7.02-7.07(\mathrm{~m}, 2 \mathrm{H}), 7.10-7.16(\mathrm{~m}, 2 \mathrm{H}), 7.18$ $7.24(\mathrm{~m}, 2 \mathrm{H}), 7.29-7.35(\mathrm{~m}, 1 \mathrm{H}), 7.38-7.42(\mathrm{~m}, 1 \mathrm{H}), 7.79(\mathrm{~d}, 1 \mathrm{H}, J=8.5$ $\mathrm{Hz}), 8.84$ (bs, $1 \mathrm{H}) .{ }^{13} \mathrm{C}-\mathrm{NMR}\left(\mathrm{CDCl}_{3}, 100 \mathrm{MHz}\right) \delta: 14.3,14.5,33.2,34.4$, $36.3,40.6,60.4,61.2,112.2,120.3,122.2,125.6,125.8,128.0,128.4$, 128.5, 131.8, 136.2, 142.4, 162.3, 172.7. GC-MS (70 eV) $m / z: 393,320,260,242,215,202,169,91$ (100). Anal. Calcd. for $\mathrm{C}_{24} \mathrm{H}_{27} \mathrm{NO}_{4}$ (393.48): C, 73.26; H, 6.92; N, 3.56. Found: C, 73.29; H, 6.94; N, 3.54 . 